\title{
INVENTARIO DE LAS ARAÑAS (ARACHNIDA: ARANEAE) DE LA RESERVA FLORÍSTICA MANEJADA LA SILLA DE ROMANO, CAMAGÜEY, CUBA
}

\author{
Alexander Sánchez-Ruiz \\ Centro Oriental de Ecosistemas y Biodiversidad (BIOECO). \\ Museo de Historia Natural Tomas Romay. José A. Saco No. 601, esquina Barnada, Santiago de Cuba \\ 90100,Cuba. alex@bioeco.ciges.inf.cu
}

\section{RESUMEN}

Se realiza un inventario rápido de las arañas de la Reserva Florística Manejada La Silla de Romano, Camagüey, Cuba. En total, se registran para el área protegida 52 especies, agrupadas en 42 géneros de 25 familias. Se registra por primera vez para el área y la provincia Camagüey, el único Ctenizidae encontrado en Cuba: Ummidia nidulans (Fabricius, 1787). Se comenta además la distribución conocida de la especie exótica invasora Cyrtophora citricola (Forskål, 1775). Se consideran objetos de conservación del área protegida las siguientes ocho especies endémicas del archipiélago cubano con ámbitos geográficos muy restringidos y posiblemente con altos requerimientos de hábitats: Corythalia squamata Bryant, 1940, Camillina rogeri Alayón, 1993, Odo sp., Modisimus sp., Neon nigriceps Bryant, 1940, Phormictopus sp., Trichopelma sp. y Ummidia nidulans; la pérdida de sus hábitats naturales resulta la principal amenaza para estas especies. Se proponen cinco recomendaciones encaminadas a proteger y estudiar los objetos de conservación seleccionados.

Palabras clave: Araneae, inventario, conservación, Cuba.

Title: Inventory of the spiders (Arachnida: Araneae) from Reserva Florística Manejada La Silla de Romano, Camagüey, Cuba.

\section{ABSTRACT}

A rapid inventory of spiders was conducted in the Reserva Florística Manejada La Silla de Romano, Camagüey, Cuba. In this protected area, 52 species belonging to 42 genera of 25 families were recorded. The only Ctenizidae found in Cuba, Ummidia nidulans (Fabricius, 1787), is recorded for the first time from the area and the Camagüey province. The known distribution of the invasive exotic species Cyrtophora citricola (Forskål, 1775) is commented. The following eight Cuban endemic species with restricted distribution and high habitat requirements are considered as conservation targets in the protected area: Corythalia squamata Bryant, 1940, Camillina rogeri Alayón, 1993, Odo sp., Modisimus sp., Neon nigriceps Bryant, 1940, Phormictopus sp., Trichopelma sp. and Ummidia nidulans; the loss of their natural habitats is the main threat to these species. Five recommendations are proposed to protect and study the conservation targets selected.

Key words: Araneae, inventory, conservation, Cuba.

\section{INTRODUCCIÓN}

Los estudios aracnológicos desarrollados en los cayos del norte del archipiélago cubano, no han tenido un carácter sistemático. Los registros de especies publicados hasta el momento son resultado de recolectas aisladas, realizadas en algunos casos por personas que visitaron estos ecosistemas con el objetivo de colectar otros grupos de animales. Sin embargo, con el 
proyecto GEF/PNUD “La Biodiversidad del Grupo Insular Sabana-Camagüey, su Protección y Uso Sostenible", se publicaron dos informes que incluyeron aportes al estudio de la fauna de arácnidos de este sistema de cayos cubanos (Alcolado et al., 1999; Rodríguez-León et al., 2007). En ambos informes se registraron para el archipiélago Sabana-Camagüey 75 especies de arácnidos, de las cuales 10 son endémicas de Cuba.

Particularmente, la fauna de arañas ha sido poco trabajada en los cayos del archipiélago cubano. Las mayores contribuciones corresponden a Giraldo Alayón García, quien ha registrado y descrito varias de las especies de estos cayos (Alayón García, 1977, 1993a, 1993b y 2002; Alayón García y Platnick, 1993). Específicamente Cayo Romano, en comparación con el resto de los cayos cubanos, es uno de los menos trabajados con relación a este grupo. No obstante, Alayón García (1993a y 2002) registran especies de arañas para este cayo en particular.

Según el Sistema Nacional de Áreas Protegidas de Cuba (CNAP, 2009), toda el área de Cayo Romano está considerada dentro de la categoría de Área Protegida de Recursos Manejados (APRM). Esta área protegida incluye a la Reserva Florística Manejada La Silla de Romano, un área con altos valores naturales e históricos. En el Plan de Manejo del APRM Cayo Romano (IES, 2001) se hace referencia a la necesidad de estudios más profundos en relación a la fauna de invertebrados del Cayo y se listan sólo 16 especies de arácnidos, de las cuales tres son endémicas.

Con este trabajo se realiza un inventario biológico de las especies de arañas presentes en la Reserva Florística Manejada La Silla de Romano; identificándose los objetos de conservación y sus principales amenazas, así como proponiéndose recomendaciones para la protección y estudio de los mismos. De esta forma se ofrece una valiosa información necesaria para el Plan de Manejo del APRM Cayo Romano.

\section{OBJETIVO}

- Realizar un inventario biológico rápido de las arañas de la Reserva Florística Manejada La Silla de Romano, Camagüey, Cuba.

\section{MATERIALES Y MÉTODOS}

La Reserva Florística Manejada La Silla de Romano (RFM) ocupa 2,141 Ha y su mayor altura es precisamente la elevación La Silla de Romano, con $62 \mathrm{msnm}$, la cual constituye una de las mayores alturas del archipiélago Sabana-Camagüey (IES, 2001). Durante seis días se realizaron recorridos por varios sitios dentro del área protegida: Las Ruinas (LR) $22^{\circ} 00^{\prime} 01^{\prime \prime} \mathrm{N}-77^{\circ} 39^{\prime} 04^{\prime \prime} \mathrm{W}$; La Pica del Gallego (PG) $22^{\circ} 00^{\prime} 43^{\prime \prime} \mathrm{N}-77^{\circ} 38^{\prime} 33^{\prime \prime} \mathrm{W}$; Punta El Inglés (PI) $22^{\circ} 03^{\prime} 25^{\prime \prime} \mathrm{N}-77^{\circ} 38^{\prime} 50^{\prime \prime} \mathrm{W}$; Playa del Muerto (PM); Punta de Piedra (PP) $22^{\circ} 02^{\prime} 03^{\prime \prime} \mathrm{N}-77^{\circ} 37^{\prime} 48^{\prime \prime} \mathrm{W}$; Vereda del Inglés (VI); El Mangal (EM) 22 $2^{\circ} 00^{\prime} 08^{\prime \prime} \mathrm{N}-77^{\circ} 39^{\prime} 33^{\prime \prime} \mathrm{W}$; Versalles (VR) 21 ${ }^{\circ} 59^{\prime} 34^{\prime \prime} \mathrm{N}-77^{\circ} 38^{\prime} 06^{\prime \prime} \mathrm{W}$. En cada sitio se revisó exhaustivamente la vegetación, el suelo, bajo piedras y corteza de troncos, en plantas epifitas y bajo plantas de Agave sp.; además, se realizó la recolecta con red entomológica en la vegetación baja. Las recolectas fueron efectuadas durante el horario diurno, aunque en algunos sitios (EM, VR, LR) se realizaron recolectas nocturnas.

Para la confección del listado de especies se tuvo en cuenta todo el material recolectado durante este estudio, así como los registros anteriores realizados para Cayo Romano. Todo el material recolectado se encuentra depositado en la colección aracnológica del Centro Oriental de Ecosistemas y Biodiversidad (BSC.AR). 


\section{RESULTADOS Y DISCUSIÓN}

Registros notables. Para la Reserva Florística Manejada La Silla de Romano, se registran con este estudio un total de 52 especies de arañas, agrupadas en 42 géneros y 25 familias; lo cual representa el $8.5 \%$ de las especies conocidas en Cuba. De estas especies, 11 resultaron endemismos del archipiélago cubano.

Es de destacar la presencia en el área de la que posiblemente sea la única especie de Ctenizidae presente en Cuba: Ummidia nidulans (Fabricius, 1787). La misma pertenece al grupo de las Mygalomorphae que construyen madrigueras en el suelo cubriendo el orificio de entrada y salida con una pequeña tapa. Un ejemplar inmaduro de esta especie fue observado en un área cercana a El Mangal dentro de la RFM. En el Caribe, el género Ummidia Thorell, 1875 solo ha sido registrado de Jamaica (Browne, 1766; Fabricius, 1787), Cuba (Rudloff, 1996) y San Vicente (Simon, 1891). Aunque se han recolectado también ejemplares de este género en La Hispaniola, los cuales se encuentran actualmente en proceso de descripción. La especie cubana es muy rara, sólo se ha recolectado en tres oportunidades, y su posición taxonómica a nivel específico aún está en dudas, por lo que su presencia en Cayo Romano adquiere notable relevancia.

Se registra también en el área la especie invasora Cyrtophora citricola (Forskål, 1775), comúnmente llamada araña parda del Mediterráneo. Esta especie ha estado colonizando en los últimos 15 años gran parte del Neotrópico muy rápidamente. En Cuba, se localizó por primera vez en las márgenes del río Taco, Municipio Baracoa, Provincia Guantánamo (Alayón García, 2003). Con posterioridad, Sánchez-Ruiz y Teruel (2006) localizaron este singular araneido en numerosas localidades que evidenciaban que su distribución en Cuba abarcaba prácticamente toda la Región Oriental del archipiélago, hasta las provincias de Granma y Holguín. Recientemente se ha colectado en Sierra de Cubitas y Cayo Romano (Martín y Sánchez-Ruiz, 2010) siendo estas las localidades más occidentales donde se ha localizado esta especie. La presencia de esta araña en los cayos de la costa norte del archipiélago nos demuestra que continúa invadiendo los ecosistemas cubanos con rumbo al occidente del país.

A pesar de las intensas búsquedas no fue posible localizar al único endémico local del área (Camillina rogeri Alayón, 1993) una especie descrita hace más de 10 años y conocida únicamente del ejemplar hembra holotipo, localizado en Canalejas, Cayo Romano (Alayón García, 1993a). Estas arañas son Gnaphosidae muy raros que por lo general habitan bajo piedras y en la hojarasca. No es una especie abundante y se sabe muy poco sobre su historia natural.

Objetos de conservación y principales amenazas. Como objetos de conservación de la Reserva Florística Manejada La Silla de Romano, se seleccionaron ocho especies endémicas presentes en el área protegida. Entre ellas cuatro especies aún sin determinar: Trichopelma sp., Phormictopus sp., Modisimus sp. y Odo sp. Estas necesitan de mayores estudios, pues podrían constituir especies nuevas para la ciencia, convirtiéndose de esa forma en endémicos locales del área. El quinto objeto de conservación es el endemismo local Camillina rogeri Alayón, 1993, especie rara que sólo se conoce de una localidad dentro de Cayo Romano. También constituyen objetos de conservación los Salticidae Corythalia squamata Bryant, 1940 y Neon nigriceps Bryant, 1940 y el Ctenizidae Ummidia nidulans (Fabricius, 1787), todas con ámbitos geográficos restringidos.

Los ocho objetos de conservación seleccionados son especies con ámbitos geográficos muy restringidos y posiblemente con altos requerimientos ambientales, por tanto la pérdida de sus hábitats naturales resulta la principal amenaza para estas especies. Trichopelma sp., Phormictopus sp., Odo sp., Camillina rogeri y Ummidia nidulans, son especies que viven en el suelo, la hojarasca y bajo piedras y troncos caídos, por lo que la erosión y desertificación del suelo podría reducir considerablemente sus poblaciones. Modisimus sp., Corythalia squamata y 
Neon nigriceps viven en la vegetación arbustiva y herbácea, en este caso la tala de la vegetación arbustiva y la alteración o modificación de las condiciones del hábitat son las principales amenazas.

Recomendaciones. 1-. Evitar la tala y hacer énfasis en la protección de la vegetación de la primera franja costera de Cayo Romano y la vegetación arbustiva en general; teniendo en cuenta la persistencia de procesos de fragmentación, la pérdida de hábitats en el Cayo y las posibilidades reales de control y restauración de estos hábitats. 2-. Tener en cuenta y estudiar los procesos geomorfológicos intensos que potencian la erosión y salinización de los suelos en Cayo Romano. Algunos de los objetos de conservación seleccionados se conocen únicamente de estos suelos. 3-. Capacitar a los técnicos que trabajan en el área protegida para que sean capaces de identificar los objetos de conservación propuestos, y sean capaces además de llevar a cabo muestreos periódicos sobre sus poblaciones, para determinar en qué estado se encuentran las mismas. 4-. Realizar estudios sobre la dinámica poblacional de al menos la especie endémica local Camillina rogeri, para determinar: (a) distribución real de las poblaciones dentro de la Reserva, (b) localización y descripción del macho (aún desconocido), y (c) los requerimientos reales de hábitats para esta especie. 5-. Realizar otros inventarios similares en diferentes momentos del año y enfocados hacia otras áreas dentro de Cayo Romano; intensificando los muestreos dentro y fuera del área protegida para determinar la distribución real de los objetos de conservación seleccionados, así como completar la lista de especies de arañas presentes en Cayo Romano y lograr un conocimiento más profundo de la distribución de las especies.

LISTA DE ESPECIES DE ARAÑAS DE LA RESERVA FLORÍSTICA MANEJADA LA SILLA DE ROMANO, CAYO ROMANO, CUBA.

Marcadas con asterisco las especies endémicas de Cuba. El Mangal (EM); Las Ruinas (LR); La Pica del Gallego (PG); Punta El Inglés (PI); Playa del Muerto (PM); Punta de Piedra (PP); Vereda del Inglés (VI); Versalles (VR).

\section{INFRAORDEN MYGALOMORPHAE}

CTENIZIDAE

1. Ummidia nidulans (Fabricius, 1787): PG

BARYCHELIDAE

2. Trichopelma sp.*:VR

THERAPHOSIDAE

3. Phormictopus sp.* : EM

4. Phormictopus cautus (Ausserer, 1875) * : LR

INFRAORDEN ARANEOMORPHAE

FILISTATIDAE

5. Kukulcania hibernalis (Hentz, 1842): VR

SCYTODIDAE

6. Scytodes fusca Walckenaer, 1837 : VR, LR, PP

PHOLCIDAE

7. Modisimus sp. * : PG, PI, VI

8. Physocyclus globosus (Taczanowski, 1874): VR 
9. Cubanops ludovicorum (Alayón, 1976) *: EM

OONOPIDAE

10. Ischnothyreus peltifer (Simon, 1891): PP, PM

\section{OECOBIIDAE}

11. Oecobius concinnus Simon, 1893: VR

\section{DEINOPIDAE}

12. Deinopis lamia MacLeay, 1839: PG

\section{ULOBORIDAE}

13. Uloborus trilineatus Keyserling, 1883: LR, PG

\section{THERIDIIDAE}

14. Achaearanea sp.: EM, LR

15. Argyrodes sp.: EM, PG

16. Latrodectus mactans (Fabricius, 1775): LR, PP, PM, EM

17. Theridion sp.1: VR

18. Theridion sp.2: LR

19. Theridion antillanum Simon, 1894: LR

20. Tidarren sisyphoides (Walckenaer, 1841): PG, PM

\section{LINYPHIIDAE}

21. Florinda coccinea (Hentz, 1850): LR

\section{TETRAGNATHIDAE}

22. Alcimosphenus licinus Simon, 1895: PI

23. Leucauge argyra (Walckenaer, 1841): LR, VR

24. Leucauge regnyi (Simon, 1897): LR, VR, PM

ARANEIDAE

25. Argiope argentata (Fabricius, 1775): PG

26. Cyclosa caroli (Hentz, 1850): VR

27. Cyclosa walckenaeri (O. P. Cambridge, 1889): VI, PI

28. Cyrtophora citricola (Forskål, 1775): PM

29. Eriophora ravilla (C. L. Koch, 1844): EM

30. Gasteracantha cancriformis (Linnaeus, 1758): LR

31. Larinia directa (Hentz, 1847): LR

\section{LYCOSIDAE}

32. Lycosa sp.1: LR, PG, PI, PM

33. Lycosa sp.2: PM, PP, PI

\section{OXYOPIDAE}

34. Peucetia viridans (Hentz, 1832): LR, VR

35. Oxyopes sp.: PG, PM

\section{ZORIDAE}

36. Odo sp.* VR

\section{CTENIDAE}

37. Ctenus sp. *: PM

38. Ctenus vernalis Bryant, 1940 *: EM

39. Cupiennius cubae Strand, 1909: EM 
ANYPHAENIDAE

40. Hibana sp.: VR, PI

GNAPHOSIDAE

41. Camillina rogeri Alayón, 1993 *: (no se colectó)

SELENOPIDAE

42. Selenops aissus Walckenaer, 1837: EM, PI

43. Selenops sp. PG

SPARASSIDAE

44. Heteropoda venatoria (Linnaeus, 1767): VR

THOMISIDAE

45. Misumenops bellulus (Banks, 1896): LR, EM

SALTICIDAE

46. Corythalia squamata Bryant, 1940 *: LR

47. Hentzia antillana Bryant, 1940: EM

48. Hentzia sp.: PG

49. Lyssomanes antillanus Peckham y Wheeler, 1889: VI

50. Menemerus bivittatus (Dufour, 1831): VR

51. Neon nigriceps Bryant, 1940*: EM

52. Phidippus regius C. L. Koch, 1846 : EM, LR

\section{AGRADECIMIENTOS}

Muchas gracias a todo el personal de la Empresa Nacional para la Protección de la Flora y Fauna de Camagüey por las facilidades logísticas brindadas para este estudio, especialmente a Maikel Borges Rodríguez. Ana Blanco Garcés y Ramón Lorenzo Pascual Pérez ayudaron en las recolectas. El Centro de Investigaciones de Medio Ambiente de Camagüey (CIMAC), apoyó en la transportación hacia Cayo Romano. Gabriel de los Santos (MNHNSD) y Giraldo Alayón $(\mathrm{MNHNCu})$ hicieron correcciones que mejoraron notablemente el manuscrito.

\section{LITERATURA CITADA}

Alayón, G. 1977. Nuevas especies de Scytodes Latreille, 1804 (Araneae: Scytodidae) de Cuba. Poeyana 177: 1-20.

Alayón García, G. 1993a. Nueva especie de Camillina (Araneae: Gnaphosidae) de Cuba. Poeyana, 434: 1-5.

Alayón García, G. 1993b. El género Barronopsis (Araneae: Agelenidae) en Cuba. Poeyana, 435: $1-16$.

Alayón García, G. 2002. Las arañas endémicas de Cuba (Arachnida: Araneae). Revista Ibérica de Aracnología 2: 1-48.

Alayón García, G. 2003. Cyrtophora citricola (Araneae:Araneidae), registro nuevo de araña para Cuba. Cocuyo, 13: 14-15.

Alayón García, G. y N. I. Platnick. 1993. Review of the Cuban Ground Spiders of the family Gnaphosidae (Araneae: Gnaphosoidea). American Mus. Novitates, 3062: 1-9. 
Alcolado, P., E. García y M. Arellano. 1999. Protección de la biodiversidad y desarrollo sostenible en el ecosistema Sabana-Camagüey. Informe de proyecto GEF/PNUD Sabana-Camagüey CUB/92/G31. La Habana, Cuba: 145 pp.

Browne, P. 1766. The Civil and Natural History of Jamaica. Printed for the author. London, $490 \mathrm{pp}$.

Centro Nacional de Áreas Protegidas (CNAP). 2009. Plan del Sistema Nacional de Áreas Protegidas 2009-2013. Escandón Impresores, España. 190 pp.

Fabricius, J. C. 1787. Mantissa insectorum sistens eorum species nuper detectas adiectis characteribus genericis, differentiis specificis, emendationibus, observationibus. Halfniae. Vol. 1. 348 pp.

Instituto de Ecología y Sistemática (IES). 2001. Plan de Manejo de Cayo Romano. La Habana, Cuba: 64 pp.

Martin-Castejón, Y. y A. Sánchez-Ruiz. 2010. Registros más occidentales de Cyrtophora citricola (Forskål, 1775) (Araneae: Araneidae) en Cuba. Novitates Caribaea 3: 83-84.

Rodríguez-León, R., I, Fernández, R. Núñez, D. Rodríguez, L. Bidart, A. Ávila, M. Hidalgo, M. López y A. Rivero. 2007. Invertebrados terrestres. Informe de proyecto PNUD/GEF Sabana-Camaguey CUB/98/G32; CUB/99/G81. (Eds.) Alcolado, P., García, E. y Arellano, M. La Habana, Cuba: 26-30.

Rudloff, J. P. 1996. Erstnachweis der Gattung Ummidia Thorell, 1879 auf Kuba (Ctenizidae: Mygalomorphae). Arthropoda 4: 48-54.

Sánchez-Ruiz, A. y R. Teruel. 2006. Acerca de la presencia de Cyrtophora citricola (Forskål, 1775) (Araneae: Araneidae) en Cuba. Boletín Sociedad Entomológica Aragonesa 38: 335336.

Simon, E. 1891. On the spiders of the island of St. Vincent. Part 1. Proc. Zool. Soc. Lond. 1891: 549-575.

[Recibido: 16 de febrero, 2011. Aceptado para publicación: 08 de febrero, 2012] 